\title{
CDISC SDTM Age Group Terminology
}

National Cancer Institute

\section{Source}

National Cancer Institute. CDISC SDTM Age Group Terminology. NCI Thesaurus. Code C66780.

Terminology codelist used with Age Group within the Clinical Data Interchange Standards Consortium Study Data Tabulation Model. 